\title{
The Research and Implementation on Pick-up Health Information and Self Health Management for Community Residents
}

\author{
Suxiang Weng ${ }^{1}$, Yixin $\mathrm{Li}^{2}$, Dequan $\mathrm{Qi}^{3}$, Chong $\mathrm{Chen}^{4}$, Runmin Dang ${ }^{5}$, Hui Gao ${ }^{6}$, Changhao Zhen ${ }^{5}$, \\ Shengji Fang, *
}

${ }^{1}$ School of Management, Jilin Medical University, Jilin, China

${ }^{2}$ Information Center, Jilin Medical University, Jilin, China

${ }^{3}$ School of Engineering, Jilin Medical University, Jilin, China

${ }^{4}$ Jilin Health Bureau, Jilin, China

${ }^{5}$ Affiliated Hospital, Jilin Medical University, Jilin, China

${ }^{6}$ School of Public Health, Jilin Medical University, Jilin, China

Email address:

214871548@qq.com (Shengji Fang)

${ }^{*}$ Corresponding author

\section{To cite this article:}

Suxiang Weng, Yixin Li, Dequan Qi, Chong Chen, Runmin Dang, Hui Gao, Changhao Zhen, Shengji Fang. The Research and Implementation on Pick-up Health Information and Self Health Management for Community Residents. Science Journal of Public Health.

Vol. 5, No. 5, 2017 pp. 401-407. doi: 10.11648/j.sjph.20170505.17

Received: June 3, 2017; Accepted: July 11, 2017; Published: August 30, 2017

\begin{abstract}
In this paper the requirement of personal information protection among the youth and middle-age people was studied through the investigation of self-health management desirability, a set of solution of self-health management by protecting personal information was brought out at the same time. By using 2000 questionnaires to investigate methods of transferring personal information, self-health management desirability among 35-50 aged people and methods of getting health instruction from practitioner, it seems that people of 35-50 aged have strong willing of self-health management, but must under the protection of personal information. The conclusion of this paper is transferring anonymous health data and getting effective health instruction meet the desirability of people, especially youth and middle-age people.
\end{abstract}

Keywords: Pick-Up Health Information, Electronic Health Records (EHR), Self Health Management

\section{Introduction}

Along with the medical care revolution in China since 2009 , the health management role of each community health service organization was put forward clearly [1]. It was considered as one of the most important step to solve many key problems of medical services [2]. In 2012 national conference of medical revolution, the increasingly important position of community health service organization was emphasized again which the main idea focused on improving the system of tiered medical services so that people having easy access to quality medical services [3].

With extensive permeation of information technology in medicine, patient-centered system will be the core of system framework in modern medical system $[4,5]$. The basic management of patient-centered is health data, with popularization of wearable devices and gradual elevations of self-health management in masses, health data are not only from hospital, and also the users have PGD (Person Generated Data). All these data record people's health index in real time, reflecting health condition to some extent, also involving possible health problems. But such as PGD motion data, experienced data, they need professional doctors to explain which make them be effective health guidance. It is a hot issue in medical institutions all around the world that how to use these collected data, providing consulting services for people, or how to combine PGD and stored data in medical institutions, being the basis of health examination [6-11]. 
Resident's electronic health data (EHR), it not limits to medical records and electronic medical records, but also contain other information generated by community health activities [12]. It is a systematic archive consists of personal basic information form, health examination form, acceptance record, consultation record, two-way referral sheet, residents' health records, etc. It is a dynamic, continuous and comprehensive record [13]. It is an important guarantee of meeting demands of "six in one" health service which refers to community prevention, medical treatment, health care, rehabilitation, health education, and birth guidance [14]. But at present, EHR only includes four types of focus group: children, maternal, the elderly and chronic diseases patients $[15,16]$. It's very hard to carry out filing work in young adults ( 35 to 50 years old), because they have some personal privacy protection reasons and low trust in community medical institutions. Due to this reason, most community health service centers gave up young adults filing, so their electronic health data can't survive. However, from the perspective of preventive medicine and the development of domestic slow disease younger, this part of the loss of files is the slow disease development, evolution, intervention and early treatment is the most meaningful data. How to effectively collect, including young adults, community residents' health records information is complete intelligent developing community health service and improve the level of residents' health foundation.

The medical service of community stays in the passive stage. Community is always the comparative geographically concentrated area. Health information provided by resident includes regional public medical information. How to dig out these valuable information and public them to the resident, to enhance regional epidemic alert and prevention ability is the responsibility of community medical institutions.

In this paper, the self-health management desirability of 35-50 aged people was selected as objective. As a result of the younger age of many diseases, especially chronic diseases, a considerable number of people of this age are in sub health status. Studying their ways of self-health management will help to raise the awareness of health management among young and middle-aged groups, which has certain social significance to improve national health level.

\section{Methods}

\subsection{To Determine Research Ideas by Investigating Present Community Health Service Situation}

Three representative types of community health service centers of a medium-sized city in northeastern China are selected and investigated. The population is about 150 thousand, with 74.3 percent regular residents. These communities have relative complete community health files. This first type is cooperation between enterprises and government. The second is a complete private community. The third one is private special hospital which has better reputation of special treatment among people. The research report is mainly about present situation of community medical service, business process, existed difficulties, requirement of community health service and the current service satisfaction, etc.

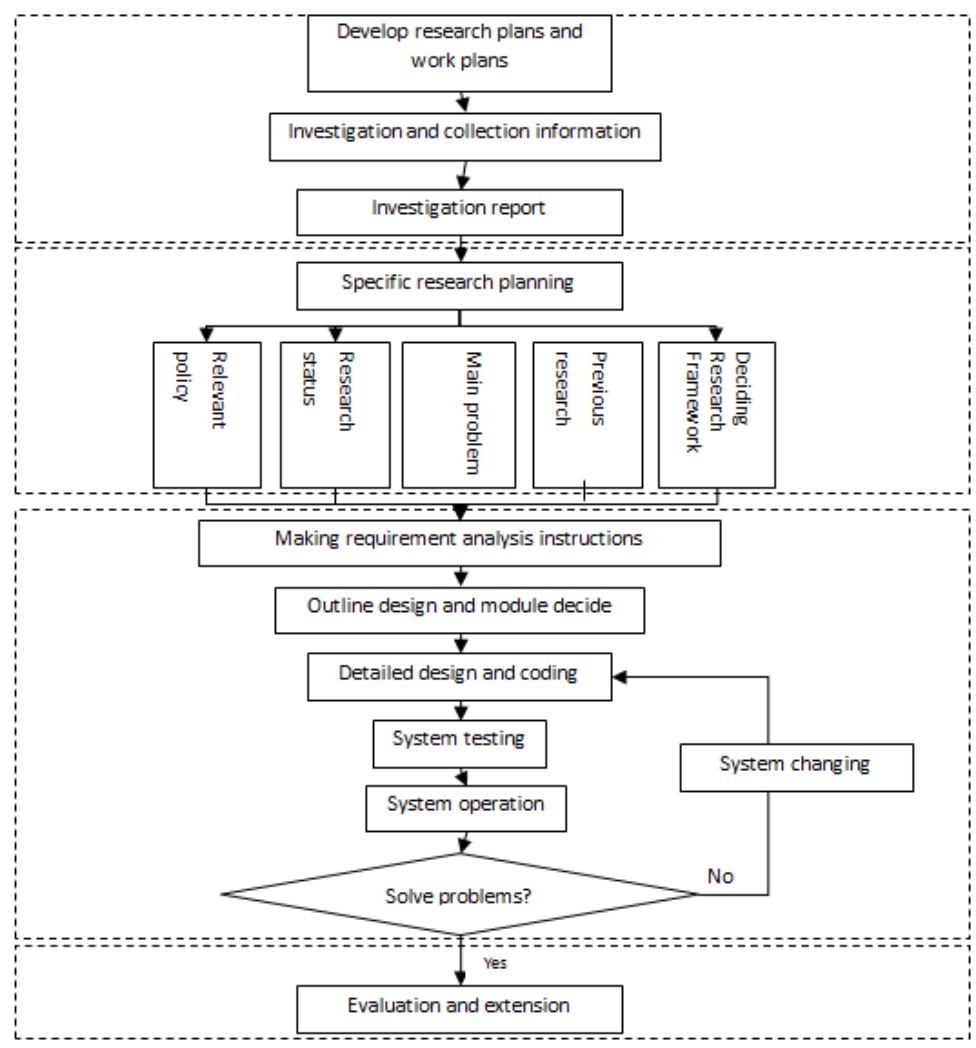

Figure 1. Research techniques routine. 


\subsection{To Confirm Technical Route with Investigation Result}

In addition to survey method, literature method, comparative method and other research methods were used to design research plan. After analysis of feasibility and risk, a scientific and reasonable technical route was designed, as showed in Figure. 1.

\subsection{To determine the Research Content by the Questionnaire of Community Residents' Demand for Community Health Service and Health Management}

With rapid development of internet and mobile internet, people have more channels for acquiring knowledge and information, including health knowledge obtaining.
Discriminating their own health condition by network is becoming people's first choice. But, meanwhile it is an obsession with leaking private information by internet. The emphasis of this thesis is how to require data in the condition of protecting personal information. How to extend the community health service through the network to every resident, so that residents can use health consulting resources safely.

Likert scale questionnaire was designed according to those problems above. Numbers 1 to 5 stands for extremely disapprove, disapprove no attitude, approve and totally approve, see Table 1. It estimates community resident's willing and method of their self-health management based on network environment.

Table 1. Basic Contents of Residents' Willingness to Health Management in Network Environment.

\begin{tabular}{|c|c|c|c|}
\hline Seq. & Health data delivery & Health knowledge acquisition & Self-health management style \\
\hline 1 & Not using network to deliver health data & Searching tools & $\begin{array}{l}\text { Check EHR In community health service } \\
\text { center }\end{array}$ \\
\hline 2 & Deliver health data anonymously & Social Media & Manage EHR by Mobile Terminal \\
\hline 3 & Using real name in network to deliver health data & Medical website & Check and management through website \\
\hline 4 & Deliver PGD in network & Network forum & Get result of data analysis \\
\hline 5 & Deliver physical examination data in network & APP & Get historical health data \\
\hline 6 & Deliver data to the website of community health service center & Text message & Get personal health instruction \\
\hline 7 & Deliver health data to any medical website & Email & Get reminder information \\
\hline
\end{tabular}

All the questionnaires were collected anonymous, given out by the community.

\section{Results}

2000 questionnaires were given out in three communities, recycled 977 effective questionnaires; the rate of recovery was $48.85 \%$. During the investigators, the proportion of male was $48.4 \%$, the proportion of female was $51.6 \%$. The structure of investigators was reasonable, as age distribution was seen as in Table 2 and education degree distribution was seen as in Table 3.

Table 2. Statistical table of age distribution.

\begin{tabular}{llllll}
\hline & & Frequency & Percentage & Effective percentage & Cumulative percentage \\
\hline & Under 35 years old & 442 & 45.2 & 45.2 & 45.2 \\
& Between 35-39 years old & 300 & 30.7 & 30.7 & 75.9 \\
Effective & Between 40-44 years old & 120 & 12.3 & 12.3 & 88.2 \\
& Between 45-49 years old & 70 & 7.2 & 7.2 & 95.4 \\
& Over 50 years old & 45 & 4.6 & 4.6 & 100.0 \\
& Totally & 977 & 100.0 & 100.0 \\
\hline
\end{tabular}

Table 3. Statistical table of education degree distribution.

\begin{tabular}{llllll}
\hline & & Frequency & Percentage & Effective percentage & Cumulative percentage \\
\hline & Grad and uper & 285 & 29.2 & 29.2 & 29.2 \\
& Undergraduate & 220 & 22.5 & 22.5 & 51.7 \\
Effective & junior college education & 199 & 20.4 & 20.4 & 72.1 \\
& High school & 174 & 17.8 & 17.8 & 89.9 \\
& Other else & 99 & 10.1 & 10.1 & 100.0 \\
& Totally & 977 & 100.0 & 100.0 \\
\hline
\end{tabular}

According to the statistical results of questionnaire, 78.7 percent of residents have consciousness of self-health management. They hope they can self-manage and track their long term health data.

Meanwhile, the Kruskal-Wallis results showed that people had obvious difference of health management conscious at different ages. The statistical quantity is $87.456, \mathrm{p}<0.001$, as in Table 4.
Table 4. Rank of every age group.

\begin{tabular}{llll}
\hline & Age & N & Average of rank \\
\hline & Under 35 years old & 442 & 406.74 \\
Health & Between 35-39 years old & 300 & 586.62 \\
management & Between 40-44 years old & 120 & 550.54 \\
consciousness & Between 45-49 years old & 70 & 519.43 \\
& Over 50 years old & 45 & 434.81 \\
& Totally & 977 & \\
\hline
\end{tabular}


The proportion of 35-50 years old people who have strong demand for self-management is 72.0 percent. It is higher than 44.8 percent of 35 years old people and 53.3 percent of 50 years old people. This shows that the younger trend of all kinds of disease forced the youth who are between 35 to 50 years old focus on their health condition. So it has great realistic significance and actual benefit for us to design a set of self-health management which aims at "I am the master of my health".

The self-health management which aims at "I am the master of my health" is a complete project including data collection and storage, data analysis and sending, health warning and guidance. The manager is community residents themselves. This set of project helps the residents to know their health condition without going out, to get professional guidance and advice.

\subsection{Pick-up Residents'Health Data Based on Privacy Protection}

Firstly, investigating residents' option of "privacy data delivery and Storage security awareness", see Table 5.

Table 5. Statistical table of privacy data delivery and Storage security awareness.

\begin{tabular}{llllll}
\hline & $\begin{array}{l}\text { Using website by } \\
\text { real name register }\end{array}$ & $\begin{array}{l}\text { Using website } \\
\text { anonymously }\end{array}$ & $\begin{array}{l}\text { Deliver personal } \\
\text { data by cell phone }\end{array}$ & $\begin{array}{l}\text { Store personal data on } \\
\text { website using real name }\end{array}$ & $\begin{array}{l}\text { Record personal data by doctors } \\
\text { of community health service } \\
\text { center }\end{array}$ \\
\hline Highly disapproval & $252(25.8 \%)$ & $82(8.4 \%)$ & $82(8.4 \%)$ & $180(18.4 \%)$ & $54(5.5 \%)$ \\
Disapproval & $507(51.9 \%)$ & $233(23.8 \%)$ & $239(24.4 \%)$ & $388(39.7 \%)$ & $171(17.5 \%)$ \\
Not care & $118(12.1 \%)$ & $195(20.0 \%)$ & $414(42.4 \%)$ & $235(24.1 \%)$ & $432(44.2 \%)$ \\
Approval & $73(7.5 \%)$ & $403(41.2 \%)$ & $211(21.6 \%)$ & $145(14.8 \%)$ & $256(26.2 \%)$ \\
Highly approval & $27(2.7 \%)$ & $64(6.6 \%)$ & $31(3.2 \%)$ & $29(3.0 \%)$ & $64(6.6 \%)$ \\
\hline
\end{tabular}

The results showed that $77.7 \%$ of the residents disapproved of real-name registration using the website or system, only $10.2 \%$ of the residents were in favor of it. But the approved proportion had risen to $47.8 \%$ who agreed to use website or system anonymously. Besides that, nearly $60 \%$ $(58.1 \%)$ residents were against saving personal information in the website. All of this showed that the protection of private information was an important prerequisite for community residents. The management of personal health files without publishing private information is the important basis for trusting community health service center.

At the same time, it was found that even the community doctors offer door-to-door service to do registration, there are also $23.0 \%$ of residents having clear disapproval opinions, and only $32.7 \%$ of residents hold approval opinions. This fact was in concert with information got by investigating the community. People do not have enough trust in community service center. They considered that the community had not guided effectively on health problem, also, people had negative attitude towards filing in community service center. Low-rate filing is the most fundamental problem in the community service center.

However, if using mobile phone as the main medium, there is no distinguishing difference between $24.8 \%$ and $32.8 \%$, which stands for pro and con. This shows that mobile internet having deep effect on people. On the other hand, it provided data support for us to develop health filing by mobile internet.

Because the network information security is the most concerned problem for everyone, so if sending someone's information to some website with promise of protecting personal privacy, the proportion of approval will rise to nearly 80 percent, see Table 6 .

Table 6. Upload personal data based on privacy protection.

\begin{tabular}{|c|c|c|c|c|c|}
\hline & & Frequency & Percentage & Effective percentage & Cumulative percentage \\
\hline \multirow{6}{*}{ Effective } & 1 & 37 & 4.3 & 4.3 & 4.3 \\
\hline & 2 & 139 & 16.2 & 16.2 & 20.5 \\
\hline & 3 & 168 & 19.5 & 19.5 & 40.0 \\
\hline & 4 & 404 & 47.0 & 47.0 & 87.0 \\
\hline & 5 & 112 & 13.0 & 13.0 & 100.0 \\
\hline & Totally & 860 & 100.0 & 100.0 & \\
\hline
\end{tabular}

(1- Highly disapproval; 2- Disapproval; 3- Not care; 4- Approval; 5- Highly approval)

Therefore, the self-health management platform designed in this paper allows user to register and use anonymously, by using several methods such as integrated application of websites, SMS, mobile phone APP to collect PGD and health check data, to complete residents EHR maintenance. As long as the anonymous user updates PGD, check data or health data, they will get analysis results automatically.

With the improving of dependency and trust, the user will demand further personalized medical treatment. There will be demand for real name transform. Once an anonymous user becomes a real-name user, there will be a professional doctor provides personalized treatment guidance and advice from the community service center.

No matter the user registers the system anonymously or with real name, they all have the rights of managing self-health file, receiving disease warning broadcast, online health index analysis, etc. The real-name registrants have rights of getting professional guidance online from exclusive doctors, chronic disease intervention online, etc. After the user complete "real-name" information and being reviewed, 
they will transfer to real-name user. This lead the user transfer slowly.

The gradual transitions from anonymous user to real-name user have two functions. On the one side, it helps the community service center complete residents' health information, enhance filing efficiency, complete EHR data system of the hospital. On the other side, it establishes an effective communicating bridge between professional doctors and resident, providing basic data support and assurance. In order to ensure data security, physical separation management was used between anonymous users and real-name users.

\subsection{Using Self-Health Consulting Service System to Get Professional Health Guidance}

Among all the surveyed people, their expectations of "how to get health guidance" showed as Figure. 2. The results showed that ubiquitous network paved a path for people to know about health and disease information. Modern men pay more attention to health problem at any time when they are at the office, at home, even on their way back home, they increasingly tend to use the advantages of internet and mobile internet.

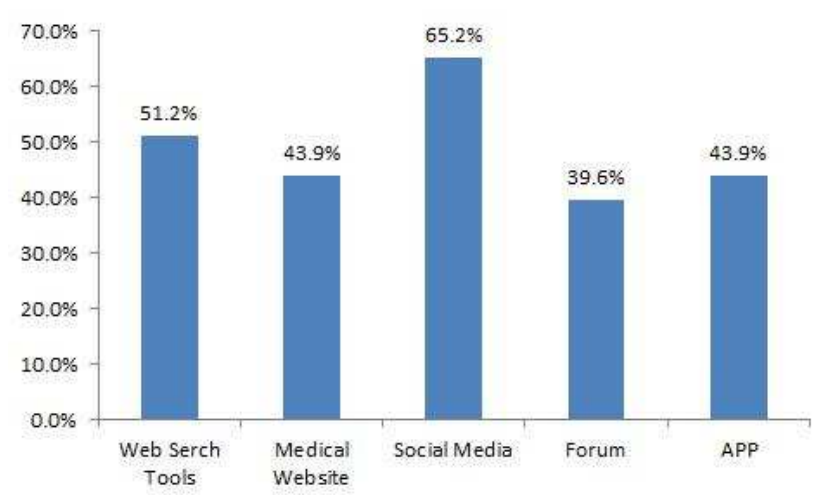

Figure 2. Willingness to obtain health instruction.

The health information got from network query often has no individual specialties. It's hard for us to identify true or false especially for various network information. It can be made sure for getting healthy, scientific, accurate and trustable only if connecting with professional doctors. It is another emphasis of this thesis to discuss how to give personalized and accurate guidance to the residents, to help them realize "I am the master of my health." It is also the emphasis of community service center.

The following Table 7 shows that 88 percent of the residents hope to get effective suggestion of health care from community service center.

Table 7. Hope to get effective suggestion of health care from community service center.

\begin{tabular}{|c|c|c|c|c|c|}
\hline & & Frequency & Percentage & Effective percentage & Cumulative percentage \\
\hline \multirow{6}{*}{ Effective } & 1 & 20 & 2.0 & 2.0 & 2.0 \\
\hline & 2 & 97 & 9.9 & 9.9 & 12.0 \\
\hline & 3 & 231 & 23.6 & 23.6 & 35.6 \\
\hline & 4 & 369 & 37.8 & 37.8 & 73.4 \\
\hline & 5 & 260 & 26.6 & 26.6 & 100.0 \\
\hline & Totally & 977 & 100.0 & 100.0 & \\
\hline
\end{tabular}

(1- Highly disapproval; 2- Disapproval; 3- Not care; 4- Approval; 5- Highly approval)

Due to the limitation of staff number, aimed at updated health data (anonymous or real name), the system will do vertical comparison with health data, condition analysis, potential health risk, and send health alert information, treatment suggestion to the user. This help the resident know their health condition and change without going out. Meantime, if the resident would like to use their real names with more trusts on community service center, there will be professional doctors who answer more complicated questions, provide one-to-one community, online $\mathrm{q} \& \mathrm{a}$, and more personalized health guidance.
The above information transmission way provides a good example for community service center to enhance health filing efficiency in the era of rapid development of mobile internet.

The statistics showed that, among the people who have willing to get professional treatment from community doctors, there is 67.6 percent of people who want to connect with community service center with mobile phone. They are mainly from 35 to 50 years old, who are familiar with mobile internet, showed in Table 8 .

Table 8. Willing to connect with community service center with mobile phone.

\begin{tabular}{|c|c|c|c|c|c|}
\hline & & Frequency & Percentage & Effective percentage & Cumulative percentage \\
\hline \multirow{6}{*}{ Effective } & 1 & 71 & 8.3 & 8.3 & 8.3 \\
\hline & 2 & 208 & 24.2 & 24.2 & 32.4 \\
\hline & 3 & 370 & 43.0 & 43.0 & 75.5 \\
\hline & 4 & 185 & 21.5 & 21.5 & 97.0 \\
\hline & 5 & 26 & 3.0 & 3.0 & 100.0 \\
\hline & Totally & 860 & 100.0 & 100.0 & \\
\hline
\end{tabular}

(1- Highly disapproval; 2- Disapproval; 3- Not care; 4- Approval; 5- Highly approval) 
With gradual transition from anonymous users to real name users, the platform not only established the effective communicative bridge between professional doctors and residents, but also helped the center to absorb PGD, construct personal health file to complete hospital EHR data system. This enhances health filing efficiency from 35 to 50 years old and makes feasible based on community, ensuring "First Contact Care of Community" as medical reformation basis.

\subsection{Non-therapeutic Intervention of the Chronic Patients}

Chronic diseases such as diabetes and cardiovascular disease has becoming one of the greatest killers threating human health. Also the incidence has showed a younger trend It is much easier to ignore the control and daily management of chronic diseases for the youth who are really busy. Once someone has been diagnosed with chronic diseases, the EHR system can carry on long-term track and intervention automatically, then it will generate quantitative analysis and qualitative analysis report, send to the patients later.

Non-therapeutic intervention of the chronic patients including: reminding physical examination regularly, vertical comparing changing trend of physical indicators with motion information provided by wearable devices, generating advices of diet and exercise, reminding the patients to get timely medical treatment whose condition have changes. Intervention is a long-term interactive behavior. Intervention process needs doctors to know the chronic diseases patients or health indicators of the potential patients. Also the doctors have to know about the attitudes of the chronic diseases patients or the potential patients. Effective intervention should be a updating, feedback and adjusting process. Therefore, effective intervention will achieve by full exploitation the system and using $\mathrm{O} 2 \mathrm{O}$ advantage in the following conditions: transmitting intervention information and data simultaneously by analyzing physical examination online, meanwhile, knowing the patients or the potential patients well and doing good communication with them offline. This function emphasizes more on "offline effective communication", only opens to "real name" users, expanding the tracked chronic disease people served by community service center, so that the system provides the function of transforming from "anonymous user" to "real name user". Also this function attracts business working people. By managing self-health in convenient way, it brings the most meaningful intervention people into professional working area.

It has a wide social significance of absorbing youth who are familiar with internet and mobile internet by collecting data anonymously, finding and interfering young chronic patients, following the trend of preventive medicine development.

\subsection{Regional Epidemics News and Health Information Publication in Community Service Center}

Community residents living in the relative concentrated area, in a certain time, the health information they sent to community service center including regional epidemics information. The horizontal analysis of health information updated by community resident will ferret out the tendency of regional health. Sending public health information through public platform helps residents to realize earlier, prevent and control epidemics developing. It is conductive to overall improvement of regional health level.

In addition to anonymous or real-name registered users, another user is community service center. In order to realize the basic medical reformation idea of "First Contact Care of Community", he registered user management can be classified not only depending on traditional regional classification, but also dividing the community into units. The administrator can broadcast information to all the users, and the community manager (always the service center staff) also can public regional health or broadcast information in his own area. This makes the accurate positioning of public health information come true, filter useless information.

\subsection{Interface Design}

At present, there is no identical data interface between EHR and HIS. This is the main reason for data discretization and information isolation. With the continuous implement of new medical reform, seamless connection between systems will become inevitable tendency. In order to realize effective abutment between the system and other resident health file system, all the data structure definition and design are refer to national standard "health file basic structure and data standard".

However, the definition of data structure in "health file basic structure and data standard" is too abstract. The code can't reflect real definition of data index. This brings great difficulty to data structure and code. In order to take into account both system exploration convenience and designing after standard, data structure interface with field structure comparison table are established. This guarantee joint between the systems with other standard system, also the developer can define system inside structure flexibly.

\section{Discussion}

35-50 year olds are refusing to set up personal health records in the community health service center, because of their busy work, lack of confidence in community hospitals, and the protection of personal privacy information, which cause data on this population's health profile are not included in health management. On the other hand, growing trend of chronic disease development is the patients becoming younger and younger, population of 35-50 years old are the most meaningful group to make disease prevention, health management and chronic disease intervention. Meanwhile, it can improve the overall level of the whole society population health.

It was found in this research that EHR filing rate of 35-50 years old people can be improved by picking up health 
information anonymously. At the same time, their concern about health can be promoted by receiving health news or transmitting physical examination data of themselves through mobile internet, which develops self-health management concept in the whole society.

However, because there is no identical data interface for domestic EHR, an inevitable defect must be faced during research. Each community service center has to format all the collected data to seamless connected with existed EHR data. In future, focusing on this problem will make a unified interface calls shielding differences of interface data format, making data be freely conversed.

\section{Conclusion}

Community health service center is the basis of medical reform to realize "First Contact Care of Community". The most difficult problem is resident have less trust on community service center which leads to unfamiliar with resident's health conditions. The medical care lost their way. On the other side, resident PGD can't be brought into electrical health file as a measure of health condition. Most resident going to bigger hospitals leads to the common phenomenon of getting treatment harder and more expensive. Those patients who have no obvious symptom or who are in incubation period always miss the best time for diagnosis and intervention.

The system upload resident PGD to community service center by health data collection under protecting personal privacy, united with EHR data registered in the hospital. By vertical analysis automatically, the system send personalized health suggestions to the resident, help them to realize self-management idea of "I am the master of my health". This established an effective communicative bridge between community service center and resident, which alleviate the problem of getting medical treatment difficult. It is an effective solution.

At the same time, along with younger trend of chronic disease, chronic diseases patients can be found and tracked, by giving them intervention suggestion through the basic medical service organization-community health service center. This plays a really important role in finding chronic disease earlier and getting treatment earlier. The advantage of community service center is geographically relative concentrated. Using that advantage, regional public healt problem can be discovered by horizontal data mining, provide first-hand information for controlling the development of public health. Also it makes great contributions to relieve of public health events spreading.

\section{Acknowledgements}

1. Project of scientific research plan of Jilin Provincial Health Bureau, ID: 2014R028.

2. Project of scientific research plan of Jilin Provincial Education Department, ID: [2016] 233.

\section{References}

[1] Zhen Li. The influence of new medical reform on community medical in China [J]. China Market, 2013(8): 45-46.

[2] Xuezhen Li, Lijuan Yang, Xianmei Wang. Establishment and management of community health records [J]. General nursing, 2009, 7(8): 2065-2066.

[3] Zhiing Zhao, Huaifu Xu. Role of informatization construction in community health care [J]. Chinese pharmaceutical industry, 2008(6): 1-2.

[4] Brian H Shirts, Loseph S Salama, Samuel J Aronson, et al. CSER and eMERGE: current and potential state of the display of genetic information in the electronic health record. Jamia, 2015, 22(6): 1231-1242.

[5] Yuan Luo, Yu Xin, Ephraim Hochberg, etc. Subgraph augmented non-negative tensor factorization (SANTF) for modeling clinical narrative text. Jamia, 2015, 22(5): 1009-1019.

[6] S Trent Rosenbloom. Person-generated health and wellness data for health care. Jamia, 2016, 23(3): 438-439.

[7] Kevin B Johnson, Barron L Patterson, Yun-xian Ho, et al. The Feasibility of text reminder to improve medication adherence in adolescents with asthma. Jamia, 2016, 23(3): 449-455.

[8] Carolyn Petersen. Patient-generated health data: a pathway to enhanced long-term cancer survivorship. Jamia, 2016, 23(3): 456-461.

[9] Elizabeth L Murnane, Dan Coseley, Pamara Chang, et al. Self-monitoring practices, attitudes, and needs of individuals with bipolar disorder: implications for the design of technologies to manage mental health. Jamia, 2016, 23(3): 477-484.

[10] Patrick C Sanger, Andrea Hartzler, Ross J Lordon, et al. A patient-centered system in a provider-centered world: challenges of incorporating post-discharge wound data into practice. Jamia, 2016, 23(3): 514-525.

[11] Lena Mamykina, Matthew E Levine, Patricia G Davidson, et al. Data-driven health management: reasoning about personally generated data in diabetes with information technologies. Jamia, 2016, 23(3): 526-531.

[12] Zhijing Zhao. Problems and Countermeasures of informatization construction in community health care $[\mathrm{J}]$. Medical information, 2011(7): 4453-4454.

[13] Meiyan Jiang. Problems and Countermeasures of establishing residents' health records [J]. Chinese medicine guide, 2013, 3(18): 690-691.

[14] Ximing Wang. Significance of establishing health records for community residents [J]. Nei Mongol Journal of Traditional Chinese Medicine, 2014, 1: 107-108.

[15] Clarissa J Diamantidis and Stefan Becker. Health Information Technology (IT) to Improve the Care of Patients With Chronic Kidney Disease (CKD) [J]. BMC Nephrol, 2014, 15: 7-8.

[16] Xiujuan Yu, Jiancheng Dong, Zhimei Zhang, etc. Study on the utilization of community health service and the satisfaction of residents in China [J]. Chinese general medicine, 2010, 13(9): 2790-2793. 